\title{
Picked Up by a Wasp? Watch Out the Spine! A Case of Multifocal Pyogenic Spondylodiscitis
}

Geniere Nigrag S*, Casazza A, Carnevale L and Ricevuti G

Emergency Medicine Resident, Intensive Care Unit, University of Pavia, Vigevano Public Hospital, Italy

\begin{abstract}
Introduction: spondylodiscitis represents $3-5 \%$ of all cases of osteomyelitis. S. aureus is isolated in $50 \%$ of cases. Mortality ranges is $0-11 \%$. Disability may occur. Diagnosis is often a challenge. Lumbar spine is preferentially affected with multifocal involvement in $4 \%$ cases. Septic shock mortality rate is high (22-76\%). Purpose: to describe a case of septic shock due to pyogenic multifocal spondylodiscitis successfully-treated in Intensive Care Unit (ICU). Methods: 63-year-old woman presented in Emergency Department complaining 7 days back pain, weakness and diarrhea. Clinical setting suggested intra-abdominal infection. Rapid hemodynamic derangement required ICU transfer to treat septic shock and multiple organ failure. Cerebrospinal fluid (CSF) analysis showed normal count cells and hyperproteinorachia. Methicillin-sensitive S. aureus (MSSA) was isolated from blood cultures and in a very low charge from CSF. In addiction $C$. difficile toxin was found. Vertebral spine Magnetic Resonance Imaging showed spondylodiscitis in multiples vertebral bodies and intervertebral disks, paravertebral and Psoas muscles abscesses. Further anamnestic information allowed identification of recent wrist wasp sting (infected by the same MSSA). In ICU, patient underwent $C$. albicans fungemia and $P$. aeruginosa sepsis. She was treated with antibiotics and we performed advanced-cardiovascular-support, protective-mechanicalventilation and continuous-venovenous-hemofiltration. Results: after 79 days, patient was transferred to rehabilitation ward and then back home. Conclusions: despite previous delay in sepsis recognition and its underlying cause, early application of intensive support and close adherence to Survival Sepsis Campaign treatment guidelines were critical issues for the survival of this unusual spondylodiscitis case.
\end{abstract}

Keywords: Spondylodiscitis; Osteomyelitis; Mortality; Multifocal

\section{Introduction}

Spondylodiscitis is an uncommon infection that represents $3-5 \%$ of all cases of osteomyelitis [1,2]. The incidence seems to be increasing in the last years as a result of higher life expectancy of older patients with chronic debilitating diseases. Spondylodiscitis is in most cases a hematogenous infection. Staphylococcus aureus is the most frequent microorganism isolated, accounting about $50 \%$ of the all cases [3]. Mortality ranges is from 0 to $11 \%$ [2]. In a significant number of cases, recrudescence, residual neurological defects or persistent pain may occur. Diagnosis is difficult and often delayed or missed due to rarity of disease and high frequency of low back pain in general population [2]. Hematogenous pyogenic spondylodiscitis affects preferentially the lumbar spine. Multifocal involvement occurs in $4 \%$ of cases $[4,5]$.

Purpose: the objectives of this report are to describe a case of a multifocal pyogenic spondylodiscitis successfully treated in Intensive Care Unit (ICU) and to review physician attempt to this rare infection.

\section{Clinical Case}

We describe a case of 63-year-old woman living in a farm situated in Lomellina's countryside, complaining 7 days back pain, weakness and diarrhea. Clinical setting suggested intra-abdominal infection. Rapid hemodynamic derangement required ICU transfer to treat septic shock and multiple organ failure. Cerebrospinal fluid (CSF) analysis showed normal count cells and hyperproteinorachia (Table 1). Methicillin-sensitive Staphylococcus aureus (MSSA) was isolated from blood cultures and, later, in a very low charge from CSF. In addiction Clostridium difficile toxin was found. There was no history of immunodeficiency or previous antibiotics therapy. Vertebral spine Magnetic Resonance Imaging (MRI) showed spondylodiscitis in multiples vertebral bodies and intervertebral discs, paravertebral and Psoas muscles abscesses (Figures 1-6). Further anamnestic information allowed identification of recent wrist wasp sting (infected by MSSA). When questioned, the daughter referred that her mother was picked

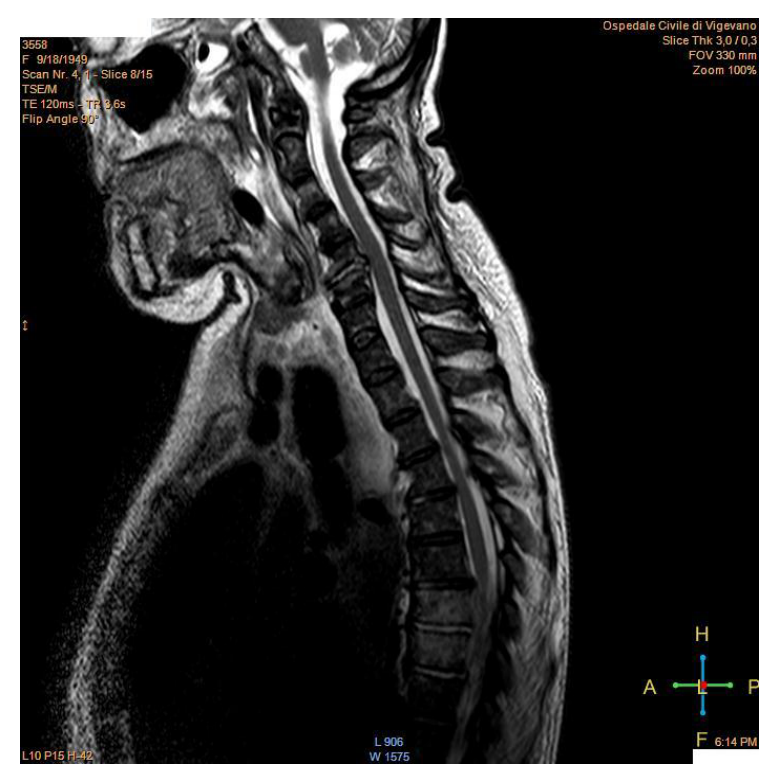

Figure 1: MRI sagittal cut showing hyperintense and inhomogeneous signal in cervical and dorsal tracts during empiric antibiotic therapy.

*Corresponding author: Geniere Nigrag S, Emergency Medicine Resident, University of Pavia, Intensive Care Unit, Vigevano Public Hospital, Italy, Tel: +39 0382 989898; E-mail: stefano.genierenigra@gmail.com

Received September 16, 2015; Accepted October 06, 2015; Published October 13,2015

Citation: Geniere Nigrag S, Casazza A, Carnevale L, Ricevuti G (2015) Picked Up by a Wasp? Watch Out the Spine! A Case of Multifocal Pyogenic Spondylodiscitis. J Clin Case Rep 5: 627. doi:10.4172/2165-7920.1000627

Copyright: (C) 2015 Geniere Nigrag S, et al. This is an open-access article distributed under the terms of the Creative Commons Attribution License, which permits unrestricted use, distribution, and reproduction in any medium, provided the original author and source are credited. 
Citation: Geniere Nigrag S, Casazza A, Carnevale L, Ricevuti G (2015) Picked Up by a Wasp? Watch Out the Spine! A Case of Multifocal Pyogenic Spondylodiscitis. J Clin Case Rep 5: 627. doi:10.4172/2165-7920.1000627

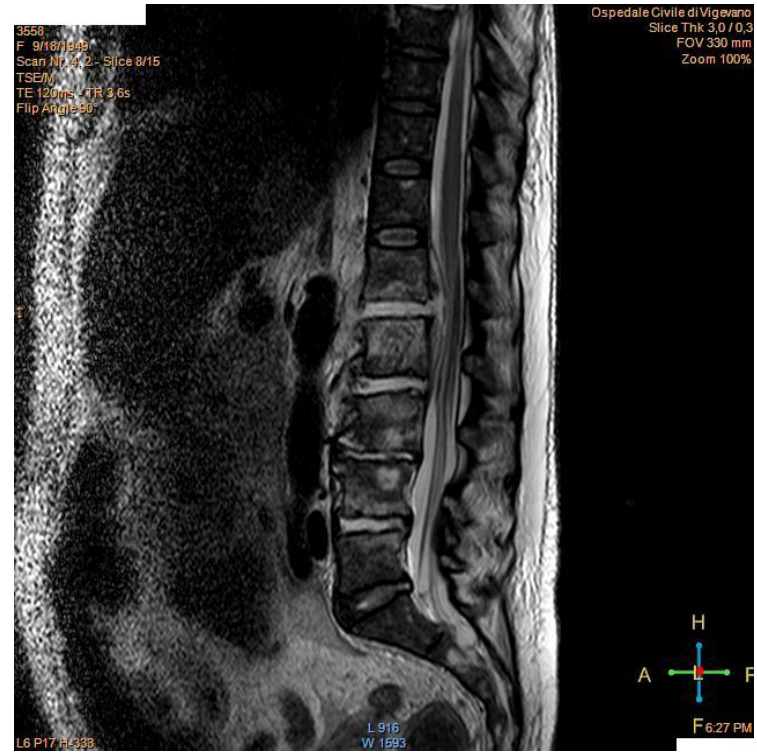

Figure 2: MRI sagittal cut showing hyperintense and inhomogeneous signal of lumbar tract during empiric antibiotic therapy.

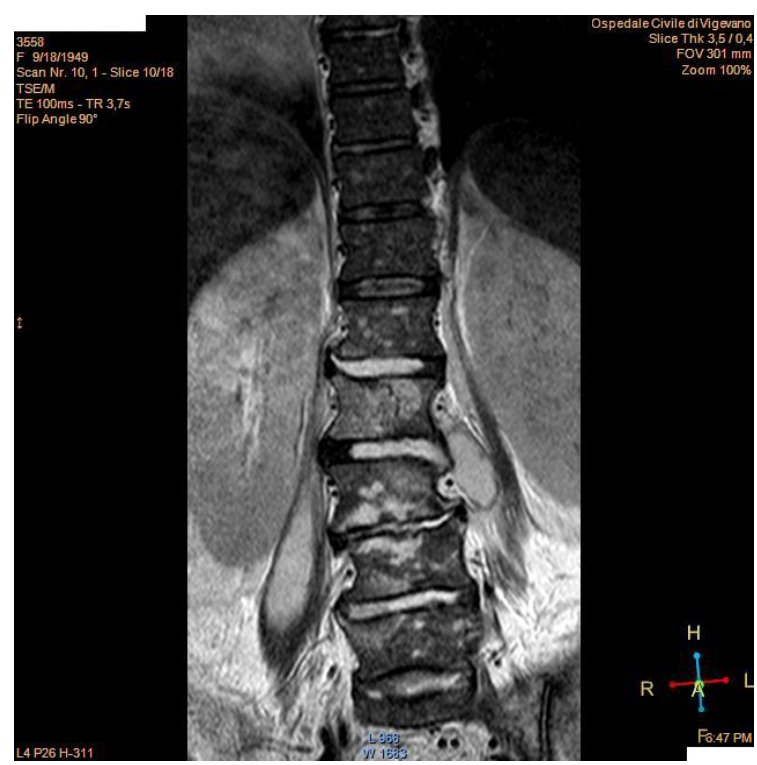

Figure 3: MRI sagittal cut showing hyperintense and inhomogeneous signal in lumbar tract and Psoas Abscess during empiric antibiotic therapy.

up by a wasp few days earlier the appearance of the back pain. In ICU, patient underwent Candida albicans fungemia and Pseudomonas aeruginosa sepsis. She was treated with antibiotics (Table 2) and we performed advanced cardiovascular support, protective mechanical ventilation and continuous veno-venous hemofiltration. After 79 days hospitalization in ICU, the patient was transferred to neurological rehabilitation in spontaneous breathing, normal renal function without signs of neurological damage. Then she went back home.

\section{Discussion}

We present a rare case of multifocal pyogenic spondylodiscitis treated in our hospital in ICU. There are two key-points about this

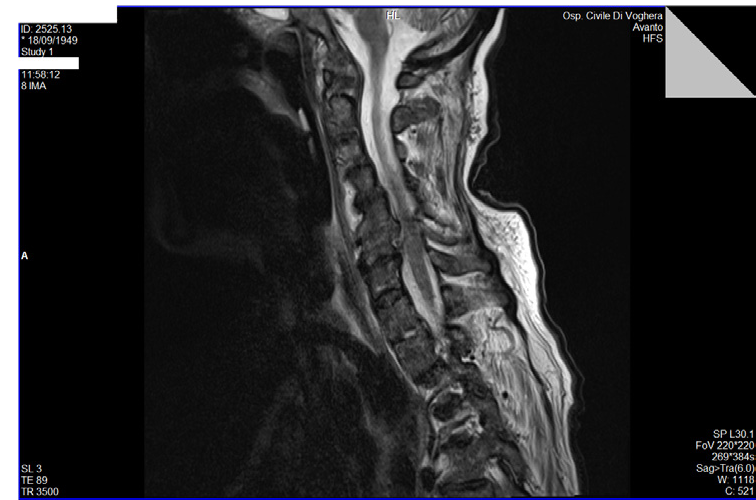

Figure 4: MRI sagittal cut showing hyperintense and inhomogeneous signal around $\mathrm{C} 7$ after 3 weeks specific antibiotic therapy antibiogram-guided.

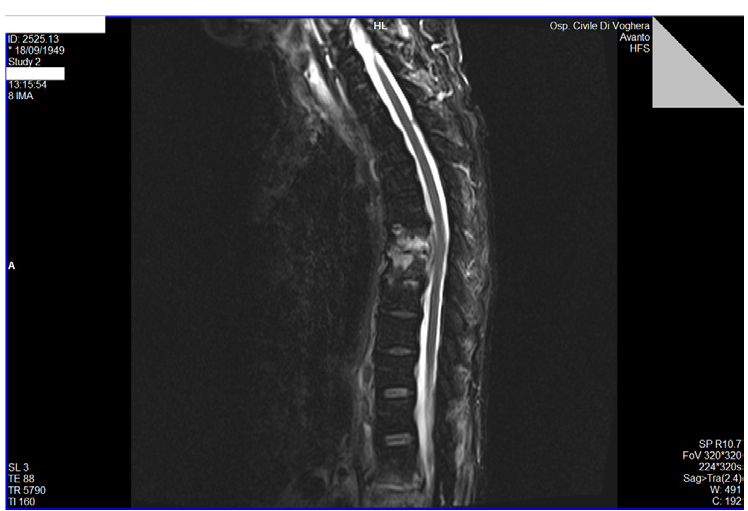

Figure 5: MRI sagittal cut showing hyperintense and inhomogeneous signal around D7 - D8 with initial medullary compression associated after 3 weeks specific antibiotic therapy antibiogram-guided.

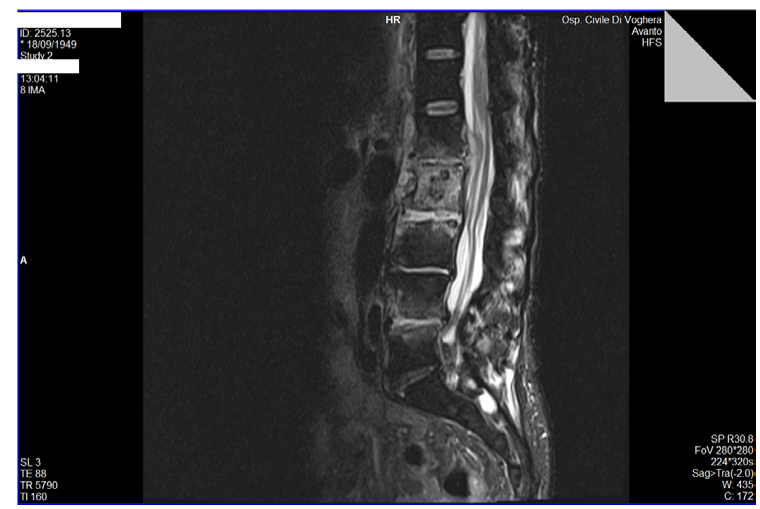

Figure 6: MRI sagittal cut showing hyperintense and inhomogeneous signal of the whole body of L2 and intersomatic discs after 3 weeks specific antibiotic therapy antibiogram-guided.

clinical case we want to discuss: firstly, the CSF analysis showed normal count cells and hyperproteinorachia. Only during the following days we documented extremely slow growth associated to a very low charge of Methicillin-sensitive Staphylococcus aureus. The interpretation of laboratory findings might be confused and misunderstood; This liquor findings are common in different situations such as contaminated fluid and autoimmune illness. The second mayor point is the importance to 
Citation: Geniere Nigrag S, Casazza A, Carnevale L, Ricevuti G (2015) Picked Up by a Wasp? Watch Out the Spine! A Case of Multifocal Pyogenic Spondylodiscitis. J Clin Case Rep 5: 627. doi:10.4172/2165-7920.1000627

Page 3 of 3

\begin{tabular}{|c|c|c|c|c|c|c|c|c|}
\hline \multicolumn{3}{|c|}{ CSF $13 / 07 / 2013$} & \multicolumn{3}{|c|}{ CSF $19 / 07 / 2013$} & \multicolumn{3}{|c|}{$\operatorname{CSF} 13 / 08 / 2013$ * } \\
\hline CSF Features & VALUE & RANGE & CSF Features & VALUE & RANGE & CSF Features & VALUE & RANGE \\
\hline Aspect & Hematic & Clear & Aspect & Turbid & Clear & Aspect & Clear & Clear \\
\hline Color & Opalescent & Colorless & Color & Dark yellow & Colorless & Color & Colorless & Colorless \\
\hline WBC & $1 / \mathrm{mmc}$ & $<5$ & WBC & $10 / \mathrm{mmc}$ & $<5$ & WBC & $1 / \mathrm{mmc}$ & $<5$ \\
\hline Proteins & $1,83 \mathrm{~g} / \mathrm{l}$ & $0,15-0,45$ & Proteins & $11,89 \mathrm{~g} / \mathrm{l}$ & $0,15-0,45$ & Proteins & $2,07 \mathrm{~g} / \mathrm{l}$ & $0,15-0,45$ \\
\hline Glucose & $72 \mathrm{mg} / \mathrm{dl}$ & $40-70$ & Glucose & 19 mg/dl & $40-70$ & Glucose & $58 \mathrm{mg} / \mathrm{dl}$ & $40-70$ \\
\hline Microscope & Red Cells ++ & & Microscope & Red Cells ++ & & Microscope & Negative & \\
\hline Colture & Negative?* & & Colture & S. aureus & & Colture & Negative & \\
\hline
\end{tabular}

Table 1: Cerebrospinal fluid findings at Day 1, day 6 and day 30. Laboratory findings show very misunderstandable results.

\begin{tabular}{|c|c|c|c|c|}
\hline \multicolumn{2}{|r|}{ ICU Infections } & \multirow[b]{2}{*}{$\begin{array}{l}0 \\
2\end{array}$} & \multicolumn{2}{|c|}{ WILD Infections } \\
\hline & & & CIPROFLOXACIN CEFTRIAXONE & Empiric (administred by other hospital) \\
\hline & & 5 & METRONIDAZOLE & C. difficile toxin + \\
\hline \multirow[t]{2}{*}{ MEROPENEM } & Pseudomonas aeruginosa (blood colture) & 10 & LINEZOLID MEROPENEM & Clinical signs of meningism \\
\hline & & 20 & CEFAZOLIN & MSSA in blood and CSF coltures \\
\hline COLISTIN (aerosol) & Multiresistant $P$ seudomonas aeruginosa (lung) & 30 & VANCOMYCIN (intrathecal) & Persistent MSSA in CSF \\
\hline ANIDULAFUNGIN & Candida albicans (blood colture) & 60 & BACTRIM MINOCYCLINE & MSSA (until hospital discharge) \\
\hline \multicolumn{5}{|c|}{ ICU days hospitalisation } \\
\hline
\end{tabular}

Table 2: Antibiotic therapy during ICU hospitalisation.

discover the main entry door of infection source. The wound due to wasp puncture was contaminated by the same MMSA. In conclusion, despite the delay in recognition of underlying causes of sepsis, early application of intensive support and close adherence to Survival Sepsis Campaign treatment guidelines were critical issues for the survival of this unusual spondylodiscitis case.

\section{References}

1. Calderon RR (1996) Overview and classification of spinal infections. Orthop Clin North Am 27: 1-8.
2. Gouliouris T (2010) Spondylodiscitis: update on diagnosis and management. J Antimicrob Chemother 65: 11-24.

3. D'agostino C (2010) A seven-year prospective study on spondylodiscitis: epidemiological and microbiological features. Infection 38: 102-107.

4. Jensen AG (1997) Increasing frequency of vertebral osteomyelitis following Staphylococcus aureus bacteraemia in Denmark 1980-1990. J Infect 34: 113118

5. Mylona E (2009) Pyogenic vertebral osteomyelitis: a systematic review of clinical characteristics. Semin Arthritis Rheum 39: 10-17. 